\title{
Factores asociados a mortalidad por neumonía en un hospital de tercer nivel de Colombia
}

\author{
Factors associated to mortality due to pneumonia \\ in a tertiary hospital of Colombia
}

\author{
Jorge Enrique Machado-Alba, Berenice Isaza, Mónica Sepúlveda • \\ Pereira (Colombia)
}

\section{Resumen}

Objetivos: determinar las variables asociadas con mortalidad por neumonía adquirida en la comunidad (NAC) en un hospital de Colombia

Materiales y métodos: estudio de corte transversal en pacientes mayores de 14 años con diagnóstico de NAC entre 1 enero 2010 y 30 marzo 2012. Se consideraron variables sociodemográficas, clínicas, paraclínicas y terapéuticas. Se hicieron análisis bivariados y multivariados con paquete estadístico SPSS-20.0

Resultados: de 297 casos con NAC, hubo predominio masculino (53.5\%), edad promedio $56.3 \pm 22.4$ años. El 22.6\% de los casos murieron. Las variables frecuencia cardiaca $>120 /$ minuto (OR:3.8; IC 95\%:1.492-10.008, $\mathrm{p}=0.005$ ), sepsis (OR:6.7; IC95\%:2.193-20.823, $\mathrm{p}=0.001$ ), ingreso a unidad de cuidado intensivo (OR:8.9; IC 95\%:2.831-28.592, $\mathrm{p}<0.001$ ) se asociaron estadísticamente con riesgo de morir por NAC.

Discusión: se identificaron los factores de riesgo asociados a mortalidad por NAC. Se debe implementar una guía de práctica clínica que cuantifique el riesgo para ofrecer la mejor atención posible y el cuidado necesario que identifique las variables relacionadas con un desenlace fatal. (Acta Med Colomb 2013; 38: xx-xx).

Palabras clave: neumonía, guías de práctica clínica, mortalidad, mortalidad hospitalaria, antibióticos, Colombia (fuente: DeCS)

\footnotetext{
Abstract

Objectives: to determine the variables associated with mortality due to Community Acquired Pneumonia ( CAP) in a hospital in Colombia

Materials and methods: cross-sectional study in patients older than 14 years with a diagnosis of CAP between January 1st, 2010 and March 30th, 2012. Socio-demographic, clinical, paraclinical and therapeutic variables were considered. Bivariate and multivariate analyzes were performed with SPSS -20.0

Results: of 297 cases with CAP there was male predominance (53.5\%); mean age $56.3 \pm 22.4$ years. $22.6 \%$ of patients died. The variables heart rate $>120 /$ minute (OR: 3.8 , 95\% CI:1.492-10.008, $\mathrm{p}=0.005)$, sepsis (OR : 6.7, 95\% CI :2.193-20.823, $\mathrm{p}=0.001)$, admission to intensive care unit (OR: 8.9, 95\% CI: $2.831-28.592, \mathrm{p}<0.001)$ were statistically associated with the risk of death by CAP.

Discussion: we identified risk factors associated with mortality due to CAP . It must implement a clinical practice guideline to quantify the risk to provide the best possible care and the care needed to identify the variables associated with a fatal outcome. (Acta Med Colomb 2013; 38: xx-xx).

Keywords: pneumonia, clinical practice guidelines, mortality, hospital mortality, antibacterial agents, Colombia (source: DeCS).
}

Dr. Jorge Enrique Machado-Alba: Grupo de Investigación Farmacoepidemiología y Farmacovigilancia, Universidad Tecnológica de Pereira-Audifarma S.A, Fundación Universitaria del Área Andina. Médico, Máster en Farmacoepidemiología Universidad Autónoma de Barcelona; Dra. Berenice Isaza-Martínez: Enfermera Epidemióloga, Fundación Universitaria del Área Andina. Hospital Universitario San Jorge de Pereira; Dra. Mónica Sepúlveda Marín Epidemióloga, Fundación Universitaria del Área Andina. Clínica Dumian. Pereira (Colombia).

Correspondencia. Dr. Jorge Enrique Machado.

E-mail: machado@utp.edu.co Recibido: 20/12/2012 Aceptado: 28/06/2013 


\section{Introducción}

La neumonía adquirida en comunidad (NAC) se define por la presencia de síntomas tales como tos, expectoración y dolor pleurítico y signos como fiebre, taquipnea y estertores crepitantes con confirmación radiológica (1). En el hemisferio norte se ha encontrado que afecta a 12 de cada 1000 personas año, en especial en las edades extremas. Más de 100 microorganismos diferentes se han identificado como posibles agentes etiológicos, siendo los más importantes Streptococcus pneumoniae, Haemophilus influenzae, Mycoplasma pneumoniae, Chlamydia pneumoniae y Moraxella catarrhalis (1-3). Algunos estudios han sugerido que el tabaquismo, alcoholismo, asma bronquial, inmunosupresión, enfermedad pulmonar, enfermedades cardiacas, cáncer, diabetes, enfermedad hepática, VIH, uso reciente de antibióticos y la edad avanzada son importantes factores de riesgo $(1,2)$.

Se ha estimado que entre el 22 y $42 \%$ de los adultos que sufren de NAC requieren hospitalización y entre el 5 y $10 \%$ requieren de atención en una unidad de cuidados intensivos (UCI) y la letalidad puede variar del 5 al $50 \%$ de los casos según la severidad del cuadro siendo más elevada en el adulto mayor y en el paciente inmunosuprimido $(3,4)$. Aunque la morbimortalidad se redujo tras la introducción de los antibióticos, el riesgo de complicaciones y muerte por NAC depende además de la edad y del estado inmune, de otros factores como la presencia de comorbilidades, el agente etiológico, la extensión de la infección pulmonar y el nivel de adherencia al manejo recomendado por las guías clínicas (5).

La definición de gravedad de la NAC permite estimar el riesgo de complicaciones y muerte del paciente, decidir los tipos de estudios complementarios que requiere (microbiológicos, radiológicos) y el lugar de la atención (ambulatorio vs hospitalario), además de planificar el tratamiento antimicrobiano empírico y su duración (6). Para sistematizar la evaluación de la gravedad de los pacientes con NAC, se han desarrollado algunos índices pronósticos tales como el PSI (pneumonia severity index, por sus siglas en inglés), el CRB65 (índice pronóstico de la Sociedad Británica de Tórax) y el SCAP (severe community acquired pneumonia, por sus siglas en inglés) con los cuales se estiman las categorías de riesgo, la severidad del cuadro y se decide el lugar y tipo de manejo $(4,7-9)$.

En Colombia faltan estudios que documenten las variables asociadas a mortalidad por NAC. Se buscó determinar los factores de riesgo sociodemográficos, clínicos, paraclínicos y de tratamiento asociados con muerte por NAC en el Hospital Universitario San Jorge (HUSJ) de Pereira entre los años 2010 y 2012, con el fin de mejorar la calidad de la atención de estos pacientes. EL HUSJ no cuenta con una guía que cuantifique el riesgo, ni la gravedad de la neumonía para orientar la conducta terapéutica. Las intervenciones para definir el riesgo de muerte por enfermedades infecciosas puede mejorar el pronóstico de los pacientes.

\section{Material y métodos}

Estudio de seguimiento de una cohorte histórica sobre la determinación de los factores de riesgo asociados con muerte por NAC en una población de pacientes mayores de 14 años de cualquier sexo, de los servicios de urgencias y hospitalización del HUSJ entre el 1 de enero de 2010 y 30 de marzo de 2012. Debe considerarse que el HUSJ es un hospital de carácter público y es el centro de referencia para el tercer nivel de atención de todo el departamento de Risaralda, Colombia. No se consideraron los pacientes que fueron atendidos en el servicio de urgencias, pero recibieron manejo ambulatorio.

Los investigadores obtuvieron la información a partir de los registros de las historias clínicas de cada caso, para lo cual se creó una base de datos donde se registraron los grupos de variables a estudio como aparece a continuación:

\section{Demográficas}

Edad, sexo, lugar de procedencia (urbana, rural)

\section{Clínicas}

1) Diagnóstico de ingreso y egreso hecho por el médico de planta del servicio o el especialista. 2) Signos y síntomas (tos, hiper o hipotermia, expectoración, dolor pleurítico, disnea, hemoptisis, compromiso del estado mental, taquicardia (>120 latidos por minuto), frecuencia respiratoria, tensión arterial, temperatura y saturación de oxígeno). 3) Comorbilidades (Enfermedad Pulmonar Obstructiva Crónica-EPOC-, Insuficiencia Cardiaca Congestiva -ICC-, Insuficiencia Renal Crónica-IRC-, Enfermedad Cerebrovascular-ECV-, Insuficiencia hepática -IH-). 4) Complicaciones: derrame pleural, síndrome de dificultad respiratoria del adulto (SDRA), sepsis, insuficiencia renal aguda (IRA). 5) Estado al egreso (vivo o fallecimiento). Se consideró únicamente el registro de fallecimiento (epicrisis y certificado de defunción) estableciese que la causa de muerte fue neumonía.

\section{Paraclínicas}

1) Conteo leucocitario; 2) Reporte de rayos $x$ de tórax; 3) Hemocultivos

\section{Tratamiento}

1) Hospitalización y días de hospitalización; 2) Ingreso a unidad de cuidado intensivo (UCI); 3) Tratamiento antibiótico empleado.

El proyecto fue sometido a revisión científicotécnica por las instancias correspondientes del HUSJ en la categoría de investigación sin riesgo, según la Resolución 8430 de 1993 del Ministerio de Salud de Colombia, que establece las normas científicas, técnicas y administrativas para la investigación en salud, fundamentados en la declaración de Helsinki.

Para el análisis de los datos, se empleó el paquete estadístico IBM SPSS Statistics 20.0 para Windows. Los estadísticos descriptivos utilizados fueron media, desviación estándar y valores máximo y mínimo para las variables 
continuas y porcentajes para las variables categóricas. Se utilizó la prueba de $X^{2}$ para la comparación de variables categóricas. Se aplicaron modelos de regresión logística binaria usando como variable dependiente la muerte durante el tratamiento por NAC y como covariables aquellas que se asociaron significativamente con la variable dependiente en el análisis bivariado. Se predeterminó el nivel de significancia estadística de $\mathrm{p}<0.05$.

\section{Resultados}

Se incluyeron todos los pacientes con diagnóstico de NAC, correspondiente a 297 casos, distribuidos entre hombres $(53.5 \%)$ y mujeres $(46.5 \%)$, con edad promedio de 56.3 \pm 22.4 años (rango: 14-97 años). Los resultados que muestran las variables sociodemográficas, clínicas y paraclínicas más importantes de estos casos se pueden observar en la Tabla 1. El 22.6\% de todos los pacientes fallecieron a causa de NAC durante el curso de la hospitalización, pero el resultado varió según el grupo de edad, siendo más bajo para los adultos entre 25 y 64 años (16.8\% de letalidad), del $17.1 \%$ para menores de 25 años y de $32.1 \%$ para mayores de 65 años. En ningún caso se estimó la gravedad del cuadro empleando escalas como PSI, CRB-65 o SCAP.

Hubo un ligero predominio de uso de antibióticos en monoterapia y algo más de la tercera parte recibieron macrólidos como parte del tratamiento (Tabla 1). Los antimicrobianos empleados por orden de frecuencia fueron ampicilina sulbactam ( $\mathrm{n}=201$ pacientes, $67.6 \%$ de los casos), claritromicina $(\mathrm{n}=110,37 \%)$, ceftriaxona $(\mathrm{n}=68,22.8 \%)$, meropenem $(n=22,7.4 \%)$, vancomicina $(n=14,4.7 \%)$, trimetoprim sulfametoxazol $(\mathrm{n}=11,3.7 \%)$, y doxiciclina $(\mathrm{n}=9$, $3 \%$ ) y otros. A $245(82.5 \%)$ pacientes se les realizó hemoleucograma, a $222(74.7 \%)$ radiografía de tórax, además se tomaron 92 hemocultivos y $8.6 \%$ reportó presencia de cocos gram positivos, $86.6 \%$ no reportó ningún microorganismo y $4.8 \%$ de casos nos llegó el reporte.

\section{Análisis bivariado}

En la Tabla 2 se presentan los resultados de los análisis bivariados que permiten comparar el subgrupo de pacientes que fallecieron a causa de NAC versus los que egresaron vivos. Se halló que las variables: edad mayor de 65 años, presencia de compromiso mental, ausencia de dolor pleurítico, taquicardia, taquipnea, saturación de oxígeno menor $90 \%$, antecedente de enfermedad cerebrovascular, desarrollo de SDRA, bacteremia, sepsis, falla renal, contar con tres factores de riesgo e ingresar a una UCI se asociaron de manera estadísticamente significativa con muerte por esta causa. Se encontró que las variables, sexo, procedencia, tiempo de hospitalización, los signos y síntomas como tos, hipertermia o hipotermia, expectoración, hemoptisis, las comorbilidades como ICC, IRC e IH, empleo de monoterapia antibiótica, uso de macrólidos no se asociaron de manera estadísticamente significativa con el riesgo de morir, a pesar de que falleció $25.1 \%$ de los pacientes que no versus $18.2 \%$
Tabla 1. Características sociodemográficas, clínicas y paraclínicas de los pacientes atendidos con diagnóstico de neumonía adquirida en la comunidad en el Hospital Universitario San Jorge de Pereira, 2010-2012.

\begin{tabular}{|c|c|c|}
\hline Características & $\begin{array}{l}\text { Número } \\
(\mathrm{n}=\mathbf{2 9 7})\end{array}$ & $\%$ \\
\hline \multicolumn{3}{|l|}{ Sociodemográficas } \\
\hline Edad (media $\mathrm{DE}^{1}$, años) & $56.3 \pm 22.4$ & \\
\hline Sexo masculino /femenino (n, \%) & $159 / 138$ & $(53.5 / 46.5)$ \\
\hline $\begin{array}{l}\text { Tiempo de hospitalización } \\
\text { (media, DE. días) }\end{array}$ & $8.9 \pm 8.8$ & \\
\hline Procedencia área urbana (n, \%) & 236 & $(79.5)$ \\
\hline \multicolumn{3}{|l|}{ Clínicas } \\
\hline Disnea & 237 & (79.8) \\
\hline Tos & 234 & $(78.8)$ \\
\hline Taquipnea & 227 & $(76.4)$ \\
\hline Fiebre & 217 & $(73.1)$ \\
\hline Dolor pleurítico & 164 & $(55.2)$ \\
\hline Expectoración & 144 & $(48.5)$ \\
\hline Temperatura axilar $<35$ o $>40^{\circ} \mathrm{C}$ & 60 & $(20.2)$ \\
\hline Taquicardia & 45 & $(15.2)$ \\
\hline Hemoptisis & 28 & $(9.4)$ \\
\hline Hipotensión arterial (<90/60mmHg) & 28 & $(9.4)$ \\
\hline Compromiso mental & 23 & (7.7) \\
\hline \multicolumn{3}{|l|}{ Paraclínicas } \\
\hline Saturación de $\mathrm{O}_{2}<90 \%$ & 107 & $(36.6)$ \\
\hline $\begin{array}{l}\text { Conteo inicial leucocitos } \\
(\text { media } \pm \mathrm{DE} ; \text { rango; leucocitos } / \mathrm{mL})\end{array}$ & $12701 \pm 6775$ & $1700-38300$ \\
\hline $\begin{array}{l}\text { Conteo control leucocitos } \\
\text { (media } \pm \mathrm{DE} ; \text { rango; leucocitos } / \mathrm{mL} \text { ) }\end{array}$ & $11607 \pm 5957$ & $1500-33600$ \\
\hline \multicolumn{3}{|l|}{ Tratamiento } \\
\hline Monoterapia antibiótica & 152 & $(51.1)$ \\
\hline Uso de macrólidos & 110 & $(37.0)$ \\
\hline \multicolumn{3}{|l|}{ Comorbilidades } \\
\hline Insuficiencia cardiaca congestiva & 76 & $(25.6)$ \\
\hline Insuficiencia renal crónica & 19 & $(6.4)$ \\
\hline Enfermedad pulmonar obstructiva crónica & 19 & $(6.4)$ \\
\hline Enfermedad cerebrovascular & 17 & (5.7) \\
\hline Cardiopatía isquémica coronaria & 9 & (3.0) \\
\hline Insuficiencia hepática & 6 & (2.0) \\
\hline Diabetes mellitus & 2 & $(0.6)$ \\
\hline Cáncer & 2 & $(0.6)$ \\
\hline \multicolumn{3}{|l|}{ Complicaciones } \\
\hline Derrame pleural & 96 & $(32.3)$ \\
\hline Síndrome de dificultad respiratoria del adulto & 94 & (31.6) \\
\hline Muerte por neumonía & 67 & $(22.6)$ \\
\hline Bacteremia, sepsis y choque séptico & 85 & $(28.2)$ \\
\hline Insuficiencia renal & 23 & (7.7) \\
\hline Ingreso a unidad de cuidados intensivos & 21 & (7.1) \\
\hline${ }^{1}$ DE: Desviación estándar & & \\
\hline
\end{tabular}


Tabla 2. Análisis bivariado de la comparación entre el desenlace muerte vs sobrevida por neumonía adquirida en la comunidad en 297 pacientes atendidos en el Hospital Universitario San Jorge de Pereira, 2010-2012.

\begin{tabular}{|c|c|c|c|c|c|c|c|}
\hline Características & $\begin{array}{c}\text { Estado al egreso vivo } \\
\text { Número }\end{array}$ & $\%$ & $\begin{array}{c}\text { Estado al egreso muerto } \\
\text { Número }\end{array}$ & $\%$ & Valor de $\mathbf{P}^{1}$ & $\mathbf{R R}^{2}$ & $\begin{array}{c}\text { I.C.95\% } \\
\text { Inf-sup }\end{array}$ \\
\hline $\begin{array}{l}\text { Con compromiso mental } \\
\text { Sin compromiso mental }\end{array}$ & $\begin{array}{c}12 \\
218\end{array}$ & $\begin{array}{l}52.2 \\
79.6\end{array}$ & $\begin{array}{l}11 \\
56\end{array}$ & $\begin{array}{l}47.8 \\
20.4\end{array}$ & 0.003 & 3.568 & $1.496-8.511$ \\
\hline $\begin{array}{l}\text { Taquicardia } \\
\text { Sin taquicardia }\end{array}$ & $\begin{array}{c}27 \\
203\end{array}$ & $\begin{array}{l}60.0 \\
80.5\end{array}$ & $\begin{array}{l}18 \\
49\end{array}$ & $\begin{array}{l}40.0 \\
19.5\end{array}$ & 0.002 & 2.762 & $1.409-5.414$ \\
\hline $\begin{array}{l}\text { Taquipnea } \\
\text { Sin taquipnea }\end{array}$ & $\begin{array}{c}167 \\
63\end{array}$ & $\begin{array}{l}73.6 \\
90.0\end{array}$ & $\begin{array}{c}60 \\
7\end{array}$ & $\begin{array}{l}26.4 \\
10.0\end{array}$ & 0.004 & 3.234 & $1.403-7.451$ \\
\hline $\begin{array}{l}\text { Saturación }<\mathbf{9 0 \%} \\
\text { Saturación }>90 \%\end{array}$ & $\begin{array}{c}74 \\
156\end{array}$ & $\begin{array}{l}69.2 \\
82.1\end{array}$ & $\begin{array}{l}33 \\
34\end{array}$ & $\begin{array}{l}30.8 \\
17.9\end{array}$ & 0.010 & 2.046 & $1.177-3.557$ \\
\hline $\begin{array}{l}\text { Enfermedad cerebrovascular } \\
\text { Sin enfermedad cerebrovascular }\end{array}$ & $\begin{array}{c}8 \\
222\end{array}$ & $\begin{array}{l}47.1 \\
79.3\end{array}$ & $\begin{array}{c}9 \\
58\end{array}$ & $\begin{array}{l}52.9 \\
20.7\end{array}$ & 0.002 & 4.306 & $1.592-11.650$ \\
\hline $\begin{array}{l}\text { Hemocultivo } \\
\text { Sin hemocultivo }\end{array}$ & $\begin{array}{c}64 \\
166\end{array}$ & $\begin{array}{l}69.6 \\
80.9\end{array}$ & $\begin{array}{l}28 \\
39\end{array}$ & $\begin{array}{l}30.4 \\
19.1\end{array}$ & 0.030 & 1.862 & $1.059-3.275$ \\
\hline $\begin{array}{l}\text { Síndrome dificultad respiratoria del adulto } \\
\text { Sin síndrome de dificultad respiratoria }\end{array}$ & $\begin{array}{c}58 \\
172\end{array}$ & $\begin{array}{l}61.7 \\
84.7\end{array}$ & $\begin{array}{l}36 \\
31\end{array}$ & $\begin{array}{l}38.3 \\
15.3\end{array}$ & $<0.001$ & 3.444 & $1.957-6.059$ \\
\hline $\begin{array}{l}\text { Bacteremia } \\
\text { Sin bacteremia }\end{array}$ & $\begin{array}{c}9 \\
221\end{array}$ & $\begin{array}{l}29.0 \\
83.1\end{array}$ & $\begin{array}{l}22 \\
45\end{array}$ & $\begin{array}{l}71.0 \\
16.9\end{array}$ & $<0.001$ & 12.005 & $5.187-27.784$ \\
\hline $\begin{array}{l}\text { Sepsis } \\
\text { Sin sepsis }\end{array}$ & $\begin{array}{c}21 \\
209\end{array}$ & $\begin{array}{l}38.9 \\
86.0\end{array}$ & $\begin{array}{l}33 \\
34\end{array}$ & $\begin{array}{l}61.1 \\
14.0\end{array}$ & $<0.001$ & 9.660 & $5.011-18.620$ \\
\hline $\begin{array}{l}\text { Falla renal } \\
\text { Sin falla renal }\end{array}$ & $\begin{array}{c}4 \\
226\end{array}$ & $\begin{array}{l}17.4 \\
82.5\end{array}$ & $\begin{array}{l}19 \\
48\end{array}$ & $\begin{array}{l}82.6 \\
17.5\end{array}$ & $<0.001$ & 22.365 & $7.280-68.703$ \\
\hline $\begin{array}{l}\text { Ingreso a unidad cuidado intensivo } \\
\text { Salas de hospitalización } \\
\text { Sin factores de riesgo } \\
\text { Un solo factor de riesgo } \\
\text { Dos factores de riesgo }\end{array}$ & $\begin{array}{c}8 \\
222 \\
28 \\
74 \\
64\end{array}$ & $\begin{array}{l}38.1 \\
80.4 \\
93.3 \\
91.3 \\
76.1\end{array}$ & $\begin{array}{c}13 \\
54 \\
2 \\
7 \\
20\end{array}$ & $\begin{array}{c}61.9 \\
19.6 \\
6.7 \\
8.7 \\
23.9\end{array}$ & $\begin{array}{c}<0.001 \\
0.028 \\
<0.001 \\
0.746\end{array}$ & $\begin{array}{l}6.681 \\
0.222 \\
0.246 \\
1.104\end{array}$ & $\begin{array}{l}2.637-16.925 \\
0.051-0.957 \\
0.107-0.564 \\
0.607-2.006\end{array}$ \\
\hline $\begin{array}{l}\text { Tres factores de riesgo } \\
\text { Joven } 14 \text { a } 24 \text { años } \\
\text { Adulto medio ( } 25 \text { a } 64 \text { años) } \\
\text { Adulto mayor ( }>65 \text { años) }\end{array}$ & $\begin{array}{c}40 \\
34 \\
107 \\
89\end{array}$ & $\begin{array}{l}62.5 \\
82.9 \\
85.6 \\
67.9\end{array}$ & $\begin{array}{c}24 \\
7 \\
18 \\
42\end{array}$ & $\begin{array}{l}37.5 \\
17.1 \\
16.8 \\
32.1\end{array}$ & $\begin{array}{l}0.001 \\
0.365 \\
0.004 \\
0.001\end{array}$ & $\begin{array}{l}2.651 \\
0.673 \\
0.422 \\
2.662\end{array}$ & $\begin{array}{l}1.448-4.853 \\
0.284-1.595 \\
0.232-0.769 \\
1.518-4.668\end{array}$ \\
\hline
\end{tabular}

de los que sí recibieron claritromicina (OR: 0.66; IC95\%: $0.368-1.190 ; \mathrm{p}=0.166$ )

\section{Análisis multivariado}

Al analizar mediante regresión logística binaria la relación entre muerte a causa de NAC y las variables independientes asociadas a ésta en los análisis bivariados, se encontró que las covariables: frecuencia cardiaca $>120 \mathrm{lpm}$, presencia de sepsis o choque séptico e ingreso a una UCI se asociaron de manera estadísticamente significativa con el riesgo de morir por NAC en el HUSJ (Tabla 3).

\section{Discusión}

La curación de la NAC es un objetivo común de clínicas y hospitales. Reducir la morbilidad, la estancia hospitalaria y sobre todo la mortalidad por NAC debe ser el propósito de toda institución que atienda pacientes con esta patología.
En este trabajo se lograron identificar los factores de riesgo asociados con mortalidad por NAC en el HUSJ. La proporción de pacientes hospitalizados que fallecen por NAC (22.6\%) es alta si la comparamos con múltiples estudios que la ubican entre el 3.7 y $16 \%(1,3,5,10-13)$ y cuando se trata de pacientes mayores de 65 años o con cuadros severos (32.1\% vs $16-38.9 \%)(1.12-13)$.

Los hallazgos relacionados con las características de los pacientes muestran diferencias en cuanto a edad, siendo nuestra población en promedio más joven que la reportada por otros autores (56.3 años vs 64.2-77.0 años) $(4,5,10)$, con predominio masculino como ha sido mostrado por otros (hombres $53.5 \%$ vs 53.9-58.0\%) $(5,10)$; y con tiempos de estancia hospitalaria similares (8.9 días vs 4.3 a 11.3 días) (5, 14). La presencia de confusión y alteración del estado mental resultó ser inferior (7.7 vs 10.7-17.3\%), pero la aparición de taquipnea (76.4 vs 12.6-21.9\%), taquicardia (15.2 vs 5.6\%), 
cardiaca, que sufren como complicación sepsis o choque séptico y que son ingresados a una UCI. Los anteriores hallazgos permiten recomendar que sea fundamental que el HUSJ implemente unas guías de práctica clínica actualizadas que cuantifiquen el riesgo con el fin de ofrecer la mejor atención posible, brindando el tratamiento antibiótico adecuado y prestando el cuidado necesario ante la aparición de las variables que se relacionan con un desenlace fatal. $\mathrm{La}$ aplicación de la guía CRB-65 ha sido capaz de predecir con bastante fiabilidad el riesgo de morir a 30 días en pacientes hospitalizados y su implementación es relativamente fácil, incluso desde un servicio de atención ambulatoria (5). Es preocupante que a pesar de la demostración con la mejor evidencia del valor que tienen los índices pronósticos, aún no sean empleados en este hospital de carácter departamental (21).

Entre las limitaciones de este trabajo se debe mencionar que no se estimó la severidad de la neumonía, por falta de una guía que la cuantifique, como tampoco se pudieron identificar los pacientes con NAC que recibieron tratamiento ambulatorio y cuál fue el desenlace en éstos. Tampoco se recogieron variables como el consumo de tabaco, alcohol, ni la etnia, que han mostrado diferencias en otros trabajos. Los resultados de esta investigación sólo pueden ser extrapolados a poblaciones que tengan similares características y que sean atendidos por hospitales de carácter público.

\section{Conflictos de Interés}

Cada uno de los autores certifica que no existen conflictos de interés, que no recibieron subvenciones, patrocinios ni otro estipendio para la investigación ni para la preparación del manuscrito. Además, ninguno ha recibido de parte de compañías farmacéuticas o de otros patrocinadores: becas, honorarios por consultorías, honorarios por participación en la revisión de los datos o en el análisis estadístico de la investigación, honorarios por escribir o revisar el manuscrito o apoyo para viajar a reuniones relacionadas con la investigación.

\section{Financiación}

Hospital Universitario San Jorge, Universidad Tecnológica de Pereira, Fundación Universitaria del Área Andina.

\section{Referencias}

1. Loeb M. Community-acquired pneumonia. Clin Evid 2010; 8: 1503.

2. Lutfiyya MN, Henley E, Chang LF, Reyburn SW. Diagnosis and treatment of community-acquired pneumonia. Am Fam Physician 2006; 73(3): 442-50.

3. Valdivia C G. [Epidemiology of community-acquired pneumonia in adults]. Rev Chilena Infectol 2005; 22 Suppl 1: s11-7.

4. McNally M, Curtain J, O'Brien KK, Dimitrov BD, Fahey T. Validity of Brit- ish Thoracic Society guidance (the CRB-65 rule) for predicting the severity of pneumonia in general practice: systematic review and meta-analysis. $\mathrm{Br} \mathrm{J}$ Gen Pract. 2010; 60(579): e423-33.

5. Saldías F, Díaz O. [Severity scores for predicting clinically relevant outcomes for immunocompetent adult patients hospitalized with community-acquired pneumococcal pneumonia]. Rev Chilena Infectol 2011; 28(4): 303-9.

6. Saldías P F, Pavié G J. [Evaluating severity of community-acquired pneumonia in adults]. Rev Chilena Infectol 2005; 22 Suppl 1: s39-45.

7. Fine MJ, Auble TE, Yealy DM, Hanusa BH, Weissfeld LA, Singer DE, et al. A prediction rule to identify low-risk patients with community-acquired pneumonia. N Engl J Med 1997; 336(4): 243-50.

8. Lim WS, van der Eerden MM, Laing R, Boersma WG, Karalus N, Town GI, et al. Defining community acquired pneumonia severity on presentation to hospital an international derivation and validation study. Thorax 2003; 58(5): 377-82

9. España PP, Capelastegui A, Gorordo I, Esteban C, Oribe M, Ortega M, et al. Development and validation of a clinical prediction rule for severe communityacquired pneumonia. Am J Respir Crit Care Med 2006; 174(11): 1249-56.

10. Myint PK, Kwok CS, Majumdar SR, Eurich DT, Clark AB, España PP, et al. The International Community-Acquired Pneumonia (CAP) Collaboration Cohort (ICCC) study: rationale, design and description of study cohorts and patients. BMJ Open 2012; 2(3): e001030.

11. Asadi L, Sligl WI, Eurich DT, Colmers IN, Tjosvold L, Marrie TJ, et al Macrolide-based regimens and mortality in hospitalized patients with communityacquired pneumonia: a systematic review and meta-analysis. Clin Infect Dis 2012; 55(3): 371-80

12. Chalmers JD, Mandal P, Singanayagam A, Akram AR, Choudhury G, Short PM, et al. Severity assessment tools to guide ICU admission in communityacquired pneumonia: systematic review and meta-analysis. Intensive Care Med 2011; 37(9): 1409-20.

13. Arnold FW, Summersgill JT, Lajoie AS, Peyrani P, Marrie TJ, Rossi P, et al. Community-Acquired Pneumonia Organization (CAPO) Investigators. A worldwide perspective of atypical pathogens in community-acquired pneumonia. Am J Respir Crit Care Med 2007; 175(10): 1086-93.

14. Siempos II, Vardakas KZ, Kopterides P, Falagas ME. Adjunctive therapies for community-acquired pneumonia: a systematic review. J Antimicrob Chemother 2008; 62(4): 661-8.

15. Bauer TT, Ewig S, Marre R, Suttorp N, Welte T. CAPNETZ Study Group. CRB-65 predicts death from community-acquired pneumonia. J Intern Med 2006; 260(1): 93-101.

16. Kruger S, Ewig S,Marre R, et al. Procalcitonin predicts patients at low risk of death from community-acquired pneumonia across all CRB-65 classes. Eur Respir J 2008; 31(2): 349-355.

17. Schaaf B, Kruse J, Rupp J. Sepsis severity predicts outcome in communityacquired pneumococcal pneumonia. Eur Respir J 2007; 30(3): 517-524

18. Schuetz P, Koller M, Christ-Crain M. Predicting mortality with pneumonia severity scores: importance of model recalibration to local settings. Epidemiol Infect 2008; 136(12): 1628-1637.

19. Myint PK, Kamath AV, Vowler SL. Severity assessment criteria recommended by the British Thoracic Society (BTS) for community acquired pneumonia (CAP) and older patients. Should SOAR (systolic blood pressure, oxygenation, age and respiratory rate) criteria be used in older people? A compilation study of two prospective cohorts. Age Ageing 2006; 35(3): 286-291.

20. Zuberi FF, Khan JA. Prospective comparison of prediction rules of mortality risk for CAP in a developing country. Int J Tuberc Lung Dis 2008; 12(4): 447-452.

21. Luque S, Gea J, Saballs P, Ferrández O, Berenguer N, Grau S. Prospective comparison of severity scores for predicting mortality in community-acquired pneumonia. Rev Esp Quimioter 2012; 25(2): 147-54. 